\title{
Epigenomics - grand challenge: much more than the developmental origins of adult health and disease
}

\author{
Michael Edward Symonds* \\ Department of Child Health, Queen's Medical Centre, University Hospital, The University of Nottingham, Nottingham, UK \\ ${ }^{*}$ Correspondence: michael.symonds@nottingham.ac.uk
}

Resetting of normal developmental pathways both before and after birth can have a diverse range of short and long term outcomes. These are dependent on the timing, duration, and magnitude of challenge to the parents and/or offspring (Symonds et al., 2010). The response, at least in the fetus, can often be organ specific and further modified by changes in the lactational, post-weaning or juvenile environment. Primary mechanisms include those related to changes in the metabolic and endocrine environment that could either be a direct consequence of epigenetic modifications or conversely contribute to these types of adaptations (McMillen et al., 2005). Given the substantial methodological challenges required to make the right measurements at the correct developmental time point(s), one expects this debate will continue in line with improvements and refinements of existing and future technologies.

Notably the question as to whether DNA methylation can actually contribute to the stabilization of gene expression patterns in differentiated mammalian tissues is controversial (Waterland et al., 2009). It is clear that the percent methylation varies substantially between genes in the same tissue and changes rapidly during development but by a much larger amount than that seen for gene expression. Furthermore, in the liver for example, changes in percent methylation with age after birth can both increase and decrease, either in parallel with changes in gene expression or in the opposite direction (Waterland et al., 2009). This reflects the complexity of the system and the likelihood of many short term responses having little long term relevance. It is therefore becoming apparent that looking at the fractional change in DNA methylation at one stage of development in a highly plastic tissue such as the liver only provides limited insight as to whether such changes can have long term consequences or are of physiological relevance when considering the main functions of that tissue in later life. Future studies are needed in which the actual degree of methylation is taken into account and whether this is really related to physiologically relevant outcomes.

It is now becoming standard practise within the study of numerous metabolic and related diseases to refer to epigenetic explanations for the different phenotype(s). For example, changing the methylation status of the diet can influence the degree of obesity in the offspring (Waterland and Jirtle, 2003). To date, the models which have been required to demonstrate initial "proof-of-principle" have not surprisingly required substantial changes in dietary composition. It is also fascinating to note that although the coat-color of the offspring can be shifted by maternal diet there is actually very little difference between the control or intervention groups in the number of offspring that are of the lean phenotype, i.e., of the pseudoagouti coat color. Other factors such as gender and transgenerational factors are therefore likely to shed further insights into this complex and challenging process (Waterland et al., 2008). Ultimately, the extent to which more subtle changes in methylation status of intervention diets can result in long term programming and related outcomes needs to be examined across a range of experimental models.

Progress in the field has not surprisingly been somewhat constrained, both by the limited amount of measurements that can be made in human cohorts of which there is still perhaps too much reliance on historical cohorts whose early life experiences were substantially different to those of the twenty-first century. Furthermore as body mass index of current populations continues to increase the relevance of data from cohorts set up even a decade ago may not be readily translated to current practices. To date, we know little about the main nutritional factors influencing body composition of the newborn and growing infant and how this may be reset by changes in either maternal body composition and/or diet. For example, extra brown ("good") fat could actually be protective against later obesity especially if it is retained into adulthood whereas additional white ("bad") fat may be preferentially lost after weaning as the infants activity increases. Long term studies that concisely examine these and related aspects of energy balance from a developmental and epigenomic perspective are clearly needed.

In animal studies it is actually quite straight forward to introduce nutritional or related challenges to the mother in order to induce intra-uterine growth retardation whereas increasing fetal growth is not as straight forward. The ability to exacerbate the adverse phenotype is also more readily apparent in small animals due in part by the normal practice of maintaining these animals within a thermoneutral environment, under constant day and night-length and fed ad libitum (Martin et al., 2010). As such a large range of outcomes appear to be much more common in small animal models (Symonds and Budge, 2009) which may be explained in part by the fact that the controls are maintained within a confined environment, are well-fed, have raised fat stores and become hypertensive (Martin et al., 2010). Consequently irrespective of the in utero environment restricting caloric intake to an amount that is closer to the animal's food intake in its natural (outdoor) environment results in raised activity, a lower body weight, less fat and lower blood pressure. Ultimately this nearly doubles lifespan (Weindruch and Sohal, 1997; Martin et al., 2010). Other factors in addition to gender that can influence the outcome include maturity at birth, litter size, species, and even strain of animal used. This may explain why transient tissue specific responses are often seen that are either unaccompanied by, or not immediately related to the major phenotypic outcome. There are clearly substantial opportunities to expand beyond "simple" nutritional based interventions in rodents maintained within a somewhat confined environment. 
One major challenge is therefore to utilize contemporary human cohorts in conjunction with relevant animal intervention studies that best represent the developmental pathways that are reset and show comparable long term adaptations. Clearly the extent to which such adaptations can result in an increased body weight and excess white fat deposition in later life is one priority area. Studies that include other important factors such as paternal age, parity, season, temperature, photoperiod etc are also needed. These may provide very different insights into epigenomic influences on development than those currently being considered. They may also be more representative of the real world rather than the more old fashioned viewpoint that it is changes between the early and later life environments that primarily contribute to long term outcomes. Apart from countries in economic transition the type of dietary environment experienced in utero is actually very similar to that in which the offspring is maintained in later life. As such programmed outcomes may not be seen when the offspring are raised on a similar diet to that experienced in utero (Symonds, 2007).

The next decade promises to be filled with excitement and continued controversy as the concepts and practical relevance of events throughout the life cycle are linked with epigenomics gain momentum and critical mass.

\section{REFERENCES}

Martin, B., Ji, S., Maudsley, S., and Mattson, M. P. (2010). "Control" laboratory rodents are metabolically morbid: why it matters. Proc. Natl. Acad. Sci. U.S.A. 107, 6127-6133.

McMillen, I. C., Adam, C. L., and Muhlhausler, B. S. (2005). Early origins of obesity: programming the appetite regulatory system. J. Physiol. 565, 9-17.

Symonds, M. E. (2007). Integration of physiological and molecular mechanisms of the developmental origins of adult disease: new concepts and insights. Proc. Nutr. Soc. $66,442-450$

Symonds, M. E., and Budge, H. (2009). Nutritional models of the developmental programming of adult health and disease. Proc. Nutr. Soc. 68, 173-178.

Symonds, M. E., Sebert, S. P., and Budge, H. (2010). Nutritional regulation of fetal growth and implications for productive life in ruminants. Animal 4, 1075-1083.
Waterland, R. A., and Jirtle, R. L. (2003). Transposable elements: targets for early nutritional effects on epigenetic gene regulation. Mol. Cell. Biol. 23, 5293-5300.

Waterland, R.A., Kellermayer, R., Rached, M. T., Tatevian, N., Gomes, M. V., Zhang, J., Zhang, L., Chakravarty, A., Zhu, W., Laritsky, E., Zhang, W., Wang, X., and Shen, L. (2009). Epigenomic profiling indicates a role for DNA methylation in early postnatal liver development. Hum. Mol. Genet. 18, 3026-3038.

Waterland, R.A., Travisano, M., Tahiliani, K. G., Rached, M. T., and Mirza, S. (2008). Methyl donor supplementation prevents transgenerational amplification of obesity. Int. J. Obes. (Lond.) 32, 1373-1379.

Weindruch, R., and Sohal, R. S. (1997). Seminars in medicine of the beth israel deaconess medical center. caloric intake and aging. N. Engl. J. Med. 337, 986-994.

Received: 22 October 2010; accepted: 27 October 2010; published online: 16 November 2010

Citation: Symonds ME (2010) Epigenomics - grand challenge: much more than the developmental origins of adult health and disease. Front. Gene. 1:1. doi: 10.3389/ fgene.2010.00001

This article was submitted to Frontiers in Epigenomics, a specialty of Frontiers in Genetics.

Copyright (C) 2010 Symonds. This is an open-access article subject to an exclusive license agreement between the authors and the Frontiers Research Foundation, which permits unrestricted use, distribution, and reproduction in any medium, provided the original authors and source are credited. 